IUHET 322

November 1995

\title{
RADIAL COULOMB AND OSCILLATOR SYSTEMS IN ARBITRARY DIMENSIONS
}

\author{
V. Alan Kostelecký and Neil Russell \\ Physics Department \\ Indiana University \\ Bloomington, IN 47405, U.S.A.
}

\begin{abstract}
A mapping is obtained relating analytical radial Coulomb systems in any dimension greater than one to analytical radial oscillators in any dimension. This mapping, involving supersymmetry-based quantumdefect theory, is possible for dimensions unavailable to conventional mappings. Among the special cases is an injection from bound states of the three-dimensional radial Coulomb system into a three-dimensional radial isotropic oscillator where one of the two systems has an analytical quantum defect. The issue of mapping the continuum states is briefly considered.
\end{abstract}




\section{Introduction}

Various types of correspondence between the Kepler-Coulomb and the isotropicoscillator systems have been extensively investigated since the influential work of LeviCivita early this century [1]. Among the correspondences of interest are mappings that can be constructed between the radial equations of the quantum systems. This subject was initiated over 50 years ago in a paper by Schrödinger [2] addressing the solution of eigenvalue problems by factorization. Schrödinger discovered a connection between the radial equation of the three-dimensional quantum Coulomb problem and the radial equation of a $D$-dimensional quantum harmonic oscillator. Using a quadratic transformation in the radial coordinate, he showed that the mapping images all the states in the three-dimensional discrete Coulomb spectrum only for oscillators with $D=2$ or 4 .

Schrödinger's idea was subsequently rediscovered or investigated by a number of authors [3, 固, 5, 6, 面]. An extension relating the radial equations of the $d$-dimensional Coulomb system and the $D$-dimensional oscillator for the special case of even dimensions $d=D$ was given in ref. [8]. A more general mapping for arbitrary $d$ and even $D$ that involves a free parameter was presented in ref. [9], along with the corresponding mappings to the supersymmetric partners of these systems. All these correspondences involve oscillators in even dimensions, and they incorporate constraints on the allowed range of angular momenta. It is possible in general to map all the states of the $d$ dimensional Coulomb system into half the states of a $D$-dimensional oscillator, where $d$ is greater than one and $D$ must be even.

Recently, it has been proposed that some restrictions on the dimensions or angular momenta can be removed with the introduction of suitable analytical deformations called quantum defects in one or both systems [10]. The motivation for this derives from the use of supersymmetric quantum mechanics [11, 12] in the context of atomic physics [13], where supersymmetry-based quantum-defect theory (SQDT) [14] provides an explicit example with direct physical relevance.

One goal of the present paper is to investigate the issue of relaxing the dimensional constraints on the radial correspondences via the introduction of analytical quantum 
defects. The treatment incorporates not only the Coulomb and oscillator systems but also their supersymmetric partners. We show that with a suitable choice of defect it is indeed possible to remove restrictions on the mappings. For instance, among the examples discussed below is a generalized mapping taking any state in the three-dimensional radial Coulomb problem into a state in an analytically modified three-dimensional radial oscillator. We also briefly consider the continuum states of the two radial systems.

The focus of this work is the set of radial correspondences as summarized above. We do not address here the different issue of obtaining surjective mappings between the full $D$-dimensional oscillator and the full $d$-dimensional Coulomb systems. This interesting question has been addressed by a number of authors, originating with the parabolic-coordinate transformation of Levi-Civita [1] that relates $D=2$ to $d=2$ and with the mapping of Kustaanheimo and Stiefel [15] in their work on celestial mechanics that relates $D=4$ to $d=3$. The latter transformation in particular has been much investigated in the quantum context [16, 17, 18, 19], and in recent years extensions connecting $D=8$ and $d=5$ have been studied [20, 21]. While more complete than the purely radial mappings, all these surjective correspondences are restricted to a narrow range of dimensions.

The organization of this paper is as follows. Section II consists primarily of background on the supersymmetric radial Coulomb and oscillator systems in arbitrary dimensions and the known correspondences between them. It contains key equations needed in the subsequent sections and provides a perspective useful for our purposes. In section III, we introduce SQDT for the radial Coulomb and oscillator systems in arbitrary dimensions, and we define mappings relating these systems to the supersymmetric sectors of the associated zero-defect cases. The general correspondence between different SQDT for the Coulomb and oscillator systems in arbitrary dimensions is established in section IV. This permits, for instance, the entire set of Coulomb radial states to be injected into a subset of the oscillator radial states for any dimensions, including odd oscillator dimensions. Section V provides a short discussion of some results arising for the continuum Coulomb states. We summarize in section VI.

To distinguish comparable quantities in the two systems, we adopt the convention 
that lower-case letters are used for Coulomb-system variables while upper-case letters are used for oscillator-system variables. An exception is made in denoting energies, for which the symbol $E$ with various sub- and superscripts is used in the Coulomb system while $F$ is used in the oscillator system.

\section{Preliminaries}

This section establishes our conventions and presents some preliminary material and results. Section IIA begins with definitions and solutions for the radial Coulomb problem in arbitrary dimensions, while section IIB similarly treats the harmonicoscillator radial problem. A one-parameter mapping between these systems is presented in section IIC. Key equations for supersymmetric quantum mechanics are given in section IID. The supersymmetric counterpart of the results in section IIA is discussed in section IIE, while that of sections IIB and IIC is covered in section IIF.

\section{IIA. Coulomb Bound States in $d$ Dimensions}

The quantum Kepler-Coulomb system in $d$ dimensions is governed by the hamiltonian

$$
h=-\frac{\hbar^{2}}{2 \mu} \nabla^{2}-\frac{\kappa}{r}
$$

where $\mu$ is the reduced mass, $\kappa$ is the force constant, and $r$ is the usual radial variable. To avoid normalization issues, we assume $d>1$. The associated radial equation is obtained from the Schrödinger equation $h \psi=E \psi$ by separating the wave function $\psi$ in generalized polar coordinates, $\psi\left(r, \theta_{1}, \ldots, \theta_{d-1}\right)=s(r) \theta\left(\theta_{1}, \ldots, \theta_{d-1}\right)$. Normalizable solutions to the ensuing radial equation are found for discrete eigenenergies given by

$$
E_{n, \gamma}=\frac{-E_{0}}{4(n+\gamma)^{2}}
$$

where $E_{0}=2 \mu \kappa^{2} / \hbar^{2}, \gamma=\frac{1}{2}(d-3)$, and $n$ is the principal quantum number taking values $n=l+1, l+2, \ldots$, with $l$ the angular-momentum quantum number arising from the separation of variables. For $d \geq 3, l=0,1,2, \ldots$ as usual. When $d=2$, the angular-momentum quantum number takes the values $0, \pm 1, \pm 2, \ldots$ In this case, we 
define the symbol $l$ to represent the modulus of the angular momentum. Note that $E_{0}$ is the magnitude of the ground-state energy in the lowest dimension considered, $d=2$.

To simplify the equations that follow, we introduce a dimensionless radial variable $y=r / r_{0}$, where $r_{0}=\hbar^{2} / 2 \kappa \mu$. It is also convenient for later considerations involving supersymmetry to work with a scaled radial function $w(y)=y^{\gamma+1} s\left(r \equiv r_{0} y\right)$, which effectively removes the first-order derivative appearing in the radial equation for $s$. The radial equation becomes

$$
\left\{-\frac{d^{2}}{d y^{2}}+\frac{(l+\gamma)(l+\gamma+1)}{y^{2}}-\frac{1}{y}-\frac{E}{E_{0}}\right\} w_{d, n, l}(y)=0 .
$$

The eigensolutions involve Sonine-Laguerre polynomials and are given by

$$
w_{d, n, l}(y)=c_{d n l} y^{l+\gamma+1} \exp \left(\frac{-y}{2(n+\gamma)}\right) L_{n-l-1}^{(2 l+2 \gamma+1)}\left(\frac{y}{n+\gamma}\right)
$$

with normalization

$$
c_{d n l}=\left[\frac{\Gamma(n-l)}{2 r_{0}^{d}(n+\gamma)^{2 l+d+1} \Gamma(n+l+d-2)}\right]^{\frac{1}{2}} .
$$

\section{IIB. Oscillator Bound States in D Dimensions}

The quantum hamiltonian for the isotropic harmonic oscillator in $D$ dimensions, $D \geq 1$, is

$$
H=-\frac{\hbar^{2}}{2 M} \nabla^{2}+\frac{1}{2} M \Omega^{2} R^{2}
$$

where $M$ is the oscillator mass, $\Omega$ is the frequency, and $R$ is the usual radial variable. Separating variables in generalized polar coordinates as before produces a radial equation that has normalizable solutions $S(R)$ for energy eigenvalues given by

$$
F_{N, \Gamma}=F_{0}(2 N+2 \Gamma+3)
$$

where $\Gamma=\frac{1}{2}(D-3), F_{0}=\frac{1}{2} \hbar \Omega$ is the ground-state energy for the lowest dimension $D=1$, and $N$ is the principal quantum number taking values $N=L, L+2, L+4, \ldots$, with $L$ the quantized angular momentum arising from the separation of variables. For 
$D \geq 3, L=0,1,2, \ldots$ as usual. For $D=2$, the angular momentum ranges over $0, \pm 1$, $\pm 2, \ldots$, and we define $L$ to be its modulus. For $D=1$, the only possibilities are $L=0$ and 1. The corresponding angular variable has two discrete values, distinguishing the two orientations of the radial vector. These two cases represent distinct radial systems for the one-dimensional oscillator, as opposed to the full one-dimensional oscillator with configuration space including both positive and negative coordinate values. For convenience in what follows, we define the parity of the radial wave functions as even if $L=0$ and odd if $L=1$.

Defining the dimensionless variable $Y=R / R_{0}$ with $R_{0}=(\hbar / M \Omega)^{\frac{1}{2}}$ and introducing for later convenience the scaled radial function $W(Y)=Y^{\Gamma+1} S\left(R \equiv R_{0} Y\right)$, the radial equation becomes

$$
\left\{-\frac{d^{2}}{d Y^{2}}+\frac{(L+\Gamma)(L+\Gamma+1)}{Y^{2}}+Y^{2}-\frac{F}{F_{0}}\right\} W(Y)=0 .
$$

The eigenfunctions are

$$
W_{D, N, L}(Y)=C_{D N L} Y^{L+\Gamma+1} \exp \left(-\frac{1}{2} Y^{2}\right) L_{\frac{N}{2}-\frac{L}{2}}^{\left(L+\Gamma+\frac{1}{2}\right)}\left(Y^{2}\right)
$$

with normalization

$$
C_{D N L}=\left[\frac{2 \Gamma\left(\frac{N}{2}-\frac{L}{2}+1\right)}{R_{0}^{D} \Gamma\left(\frac{N}{2}+\frac{L}{2}+\frac{D}{2}\right)}\right]^{\frac{1}{2}} .
$$

With our definitions for $L$ above, these expressions hold for all integral $D \geq 1$. Note that the $D=1$ normalization coefficients differ from the canonical ones by a factor of $\sqrt{2}$ because the above construction produces a normalization on the half line only.

\section{IIC. Mappings between the Coulomb and Oscillator Problems}

The wave function (4) can be mapped to the wave function (9) through the quadratic transformation

$$
Y^{2}=\frac{y}{(n+\gamma)}
$$

This correspondence also interconnects the differential equations (3) and (8). The explicit relation between eigensolutions is

$$
W_{D, N, L}(Y)=K_{d n \lambda} Y^{-\frac{1}{2}} w_{d, n, l}\left((n+\gamma) Y^{2}\right)
$$


where the quantity $K_{d n \lambda}$ maintains the normalization of the wave functions and is given by

$$
K_{d, n, \lambda}=\frac{(2 n+d-3) r_{0}^{d / 2}}{R_{0}^{d-1-\lambda}} .
$$

The quantity $\lambda$ provides an extra degree of freedom in the mapping.

The ensuing relationships among the dimensionalities and the quantum numbers of the two systems are [9]

$$
\begin{aligned}
& D=2 d-2-2 \lambda \\
& N=2 n-2+\lambda, \\
& L=2 l+\lambda .
\end{aligned}
$$

The last of these equations constrains $\lambda$ to be integral. It then follows from the first equation that this mapping has image only in the oscillators of even dimension $D$.

For given angular momenta $l$ and $L$, the relation (14) between the principal quantum numbers ensures that the stack $n \geq l+1$ of Coulomb states is in one-to-one correspondence with the stack $N \geq L$ of oscillator states, with ground states coinciding. This relation between $N$ and $n$ determines a condition relating the energies $E$ and $F$ of the two systems:

$$
\frac{F_{N, \Gamma}}{F_{0}}=2 \sqrt{\frac{E_{0}}{-E_{n, \gamma}}} .
$$

The factor of two can be viewed as originating from the scaling of $N$ relative to $n$ in the second equation in (14). Absorbing it in the definitions of $E_{0}$ or $F_{0}$ would change equations (3) or (8).

Condition (14) shows that successive Coulomb angular momenta $l$ map to every second oscillator angular momentum $L$. The entire set of radial states $|n, l\rangle$ of the $d$-dimensional Coulomb system can be mapped into a subset of the states $|N, L\rangle$ of the $D$-dimensional oscillator provided $D$ satisfies $2 \leq D \leq 2 d-2$. For even or odd $\lambda$, the mapping is then an isomorphism to even or odd $L$, respectively. For given $d$, the allowed values of the pair $(D, \lambda)$ characterizing this mapping are distinct: $(2, d-2)$, $(4, d-3), \ldots,(2 d-4,1),(2 d-2,0)$. We recover in this way Schrödinger's result that all states of the three-dimensional Coulomb system can be mapped only into 
oscillators of dimension two or four. Note that if instead $l$ is taken to be fixed, so that only a subset of states is imaged, then the allowed range of $D$ is $\llbracket 4 \mid-2 \leq 2 d-2+4 l$.

\section{IID. Supersymmetric Quantum Mechanics}

For the purposes of the present paper, only a few of the basic results of supersymmetric quantum mechanics are needed. We restrict our attention to systems with a quantum-mechanical hamiltonian $H_{S}$ and two supersymmetry charges $Q$ and $Q^{\dagger}$, obeying the defining relations of the superalgebra $\operatorname{sqm}(2)$ :

$$
\left\{Q, Q^{\dagger}\right\}=H_{S} \quad, \quad\left[Q, H_{S}\right]=\left[Q^{\dagger}, H_{S}\right]=0
$$

The representation of this algebra relevant here is two dimensional and may be parametrized as 11, 12]

$$
Q=\left(\begin{array}{cc}
0 & 0 \\
A & 0
\end{array}\right) \quad, \quad Q^{\dagger}=\left(\begin{array}{cc}
0 & A^{\dagger} \\
0 & 0
\end{array}\right) \quad, \quad H_{S}=\left(\begin{array}{cc}
H^{+} & 0 \\
0 & H^{-}
\end{array}\right)
$$

There are two component hamiltonians in this system and two associated Hilbert spaces. If the bosonic hamiltonian $H^{+}$acts on wave functions $\psi^{+}$, while the fermionic hamiltonian $H^{-}$acts on $\psi^{-}$, then the corresponding Schrödinger equations can be written

$$
H^{ \pm} \psi^{ \pm}=\left[-\frac{d^{2}}{d y^{2}}+V^{ \pm}(y)\right] \psi^{ \pm}=E^{ \pm} \psi^{ \pm}
$$

where $A$ is the operator $A=-i \partial_{y}-i U^{\prime}$ and where the supersymmetric partner potentials are defined by $V^{ \pm}(y)=U^{\prime 2} \mp U^{\prime \prime}$, with $U^{\prime}=\partial_{y} U(y)$ a specified function called the superpotential.

The ground state of a supersymmetric system lies in the bosonic sector and has zero energy. Every state in the bosonic sector other than the ground state is degenerate with a distinct state in the fermionic sector, and the operators $Q, Q^{\dagger}$ map between these paired states.

\section{IIE. Supersymmetric Coulomb System}

To construct the supersymmetric Coulomb system, the bosonic-sector combination $H^{+}-E^{+}$from Eq. (18) is identified with the radial equation (3). In the latter, a 
suitable constant must be added to the energy eigenvalues and incorporated in the potential to ensure that the ground-state energy is zero. Thus, the eigenvalues are

$$
E_{n l}^{+}=\frac{1}{4(l+1+\gamma)^{2}}-\frac{1}{4(n+\gamma)^{2}}
$$

and the bosonic potential function is

$$
v^{+}(y)=\frac{(l+\gamma)(l+\gamma+1)}{y^{2}}-\frac{1}{y}+\frac{1}{4(l+1+\gamma)^{2}}
$$

The last term in Eq. (20) is the energy shift ensuring a zero ground-state energy. Since it must be constant, $l$ must be fixed to define a supersymmetric partner.

The superpotential is specified by the function [13

$$
u(y)=\frac{y}{2(l+\gamma+1)}-(l+\gamma+1) \ln y \quad .
$$

The fermionic hamiltonian and hence the associated fermionic radial equation can then be calculated as

$$
\left\{-\frac{d^{2}}{d y^{2}}+\frac{\left(l^{\prime}+\gamma\right)\left(l^{\prime}+\gamma+1\right)}{y^{2}}-\frac{1}{y}+\frac{1}{4\left(l^{\prime}+\gamma\right)^{2}}\right\} w^{-}(y)=E_{n^{\prime} l^{\prime}}^{-} w^{-}(y)
$$

where $l^{\prime}=l+1$ and $n^{\prime}$ takes on all values of $n$ except the lowest one, $l+1$. The fermionic wave functions $w^{-}(y)$ have the same functional form as the bosonic wave functions $w^{+}(y) \equiv w(y)$ given in Eq. (4), but with $n$ and $l$ replaced by $n^{\prime}$ and $l^{\prime}$ :

$$
w_{d, n^{\prime}, l^{\prime}}^{-}(y)=w_{d, n^{\prime}, l^{\prime}}(y)
$$

The two sets of eigenvalues are degenerate for $n^{\prime}=n$ : $E_{n^{\prime}=n, l^{\prime}}^{-}=E_{n l}^{+}$.

With fixed $l$, the bosonic stack of eigenstates in order of increasing energy consists of the series of kets $|n=l+1, l\rangle,|n=l+2, l\rangle, \ldots$, with lowest energy zero. The associated fermionic stack has angular momentum greater by one unit, and starts with lowest energy corresponding to the second state of the bosonic sector: $\left|n^{\prime}=l+2, l+1\right\rangle,\left|n^{\prime}=l+3, l+1\right\rangle, \ldots$ The use of $n^{\prime}$ here is consistent with spectroscopic notation. For example, when the s orbitals of lithium are interpreted as the supersymmetric partner of the hydrogen atom, $n^{\prime}=2$ corresponds to the ground state, as expected. 
A useful one-to-one correspondence between these two stacks identifies the lowest states with each other, and successively higher states of the bosonic sector with successively higher states of the fermionic sector. It is defined by the following replacements in the bosonic wave function:

$$
\begin{aligned}
& n \longmapsto n^{\prime}=n+1 \\
& l \longmapsto l^{\prime}=l+1
\end{aligned}
$$

This stack correspondence relates eigenstates with different eigenvalues. Along with similar stack correspondences defined below, it plays a useful role in the analyses to follow.

\section{IIF. Supersymmetric Oscillator and Composition Mapping}

The bosonic component of the supersymmetric oscillator can be obtained from the radial equation (8) under a suitable energy shift. The eigenvalues are

$$
F_{N L}^{+}=2(N-L)
$$

The superpotential is specified via the function

$$
U(Y)=\frac{1}{2} Y^{2}-(L+\Gamma+1) \ln Y
$$

which generates the fermionic equation

$$
\left\{-\frac{d^{2}}{d Y^{2}}+\frac{\left(L^{\prime}+\Gamma\right)\left(L^{\prime}+\Gamma+1\right)}{Y^{2}}+Y^{2}-\left(2 L^{\prime}+2 \Gamma-1\right)\right\} W^{-}(Y)=F_{N^{\prime} L^{\prime}}^{-} W^{-}(Y)
$$

where $L^{\prime}$ is defined by $L^{\prime}=L+1$. The principal quantum number $N^{\prime}$ takes the values $N^{\prime}=L^{\prime}, L^{\prime}+2, L^{\prime}+4, \ldots$. The fermionic wave functions $W^{-}(y)$ have the same functional form as the bosonic wave functions $W^{+}(Y) \equiv W(Y)$ of Eq. (9), with $N, L$ replaced by $N^{\prime}, L^{\prime}$, respectively:

$$
W_{D, N^{\prime}, L^{\prime}}^{-}(Y)=W_{D, N^{\prime}, L^{\prime}}(Y)
$$

The fermionic energies $F_{N^{\prime} L^{\prime}}^{-}=2\left(N^{\prime}-L^{\prime}+2\right)$ are degenerate with the bosonic energies for $N^{\prime}+1=N, F_{N^{\prime} L^{\prime}}^{-}=F_{N=N^{\prime}+1, L}^{+}$. 
In order of increasing energy, the bosonic stack with fixed $L$ consists of the kets $|N=L, L\rangle,|N=L+2, L\rangle,|N=L+4, L\rangle, \ldots$, with lowest energy zero. The associated fermionic stack has angular momentum one unit greater and contains the kets $\left|N^{\prime}=L^{\prime}=L+1, L+1\right\rangle,\left|N^{\prime}=L+3, L+1\right\rangle, \ldots$

The $D=1$ case is unusual and warrants special attention. As mentioned above, the 'angular momentum' $L$ for the one-dimensional oscillator takes the values zero and one, corresponding to even and odd parity. The system resembles a single stack, but is composed of two interlocking substacks. As a result, the spacing between neighboring eigenvalues is half its value in higher dimensions. Also, in constructing the supersymmetric partner, the energy shift is $L$ dependent. Consequently, distinct shifts appear for each substack. The formalism thus establishes two independent supersymmetries, each of which respects the parity and only one of which may be considered at a time. These supersymmetries for the one-dimensional radial oscillator differ from the usual one for the full one-dimensional oscillator, where a single energy shift is effected and states of opposite parity are degenerate under the supersymmetry.

In later sections, for reasons that emerge from the construction of the generalized mapping, it is more natural to focus on the supersymmetric partner of the fermionic oscillator rather than the fermionic oscillator itself. This system, which we call 'second fermionic,' has wave functions $W_{\bar{D} N^{\prime \prime} L^{\prime \prime}}$ given by

$$
W_{D N^{\prime \prime} L^{\prime \prime}}^{=}(Y)=W_{D, N^{\prime \prime}, L^{\prime \prime}}(Y)
$$

Here, $L^{\prime \prime}$ is defined by $L^{\prime \prime}=L^{\prime}+1$, and $N^{\prime \prime}$ takes values $L^{\prime \prime}, L^{\prime \prime}+2, L^{\prime \prime}+4, \ldots$ The differential equation for this system has the same functional form as the fermionic equation (27), except for the replacement of $N$ and $L$ with $N^{\prime \prime}$ and $L^{\prime \prime}$, respectively.

The oscillator bosonic sector may be put into one-to-one correspondence with the second-fermionic sector by making the following replacements in the bosonic wave function:

$$
\begin{aligned}
N & \longmapsto N^{\prime \prime}=N+2 \\
L & \longmapsto L^{\prime \prime}=L+2 .
\end{aligned}
$$

By composition of this mapping and the ones given in sections IIC and IIE, a 
correspondence may be established between the fermionic sector of the Coulomb system and the second-fermionic sector of the oscillator. It is given by

$$
\begin{aligned}
W_{D, N^{\prime \prime}, L^{\prime \prime}}^{=}(Y) & =K_{d, n^{\prime}, \lambda} Y^{-\frac{1}{2}} w_{d n^{\prime} l^{\prime}}^{-}\left(\left(n^{\prime}+\gamma\right) Y^{2}\right), \\
Y^{2} & =\frac{y}{\left(n^{\prime}+\gamma\right)}, \\
N^{\prime \prime} & =2 n^{\prime}-2+\lambda \\
L^{\prime \prime} & =2 l^{\prime}+\lambda .
\end{aligned}
$$

The dimensions are still related as in Eq. (14). See Figure 1. We emphasize that this commutative diagram involves mappings different from those presented in ref. [9], where the second-fermionic sector is not considered.

\section{Generalized Supersymmetry-Based Quantum-Defect Theory}

In this section, we introduce analytical SQDT for the Coulomb and oscillator systems in arbitrary dimensions. From the present perspective, the goal is to obtain effective radial equations that offer sufficient flexibility to obviate the dimension and angular-momentum constraints of the usual mappings, while maintaining eigensolutions with analytical structure comparable in simplicity to those of the Coulomb and oscillator systems.

The existence of suitable deformations of the Coulomb and oscillator systems satisfying these criteria is by no means apparent a priori. In what follows, we take as a guide the SQDT that is known to provide a useful analytical description of the valence structure of physical atoms in terms of an effective one-particle radial equation [14]. This model determines an effective radial potential modifying the three-dimensional radial Coulomb equation. It generates solutions with physical eigenvalues given by the Rydberg expression

$$
E_{n^{*}}=-E_{0} / 4 n^{* 2} .
$$

Here, $n^{*}$ is the principal quantum number modified by subtracting the quantum defect $\delta$, which in general depends on the angular momentum and the principal quantum number. In section IIIA, we generalize this model to the $d$-dimensional situation. For simplicity, we take $\delta$ and its generalization in arbitrary dimensions to be independent 
of the principal quantum number. This approximation is excellent in, for example, real alkali-metal atoms [22].

Similar ideas can be implemented for the radial equation of the $D$-dimensional oscillator. The resulting oscillator SQDT are presented in section IIIB. A possible physical application of these oscillator models is to the description of a valence particle in geonium atoms formed by a group of charged particles bound in a Penning trap [10].

\section{IIIA. Generalized SQDT for the Coulomb System}

Given the $d$-dimensional Coulomb radial equation (3) with fixed angular momen$\operatorname{tum} l$, we seek to implement two modifications via an effective potential $v_{\text {eff }}(y)$ added to the left-hand side. The first desired modification is a shift in dimension, from $d$ to $d^{*}=d+j$, where $j$ is an integer that in principle could depend on $l$. We require $d^{*}>1$, so $j$ must satisfy $j>1-d$. The second desired modification is a shift in energy eigenvalues from $E_{n, \gamma}$ in Eq. (2) to the $d^{*}$-dimensional extension of the Rydberg series (see Eq. (41) below). We want both these changes to be implemented while maintaining analytical eigenfunctions with form similar to those in Eq. (4).

Remarkably, these goals can be accomplished with a relatively simple effective potential, given by

$$
v_{\mathrm{eff}}(y)=\frac{(n+\gamma)^{2}-\left(n^{*}+\gamma^{*}\right)^{2}}{4(n+\gamma)^{2}\left(n^{*}+\gamma^{*}\right)^{2}}+\frac{\left(l^{*}+\gamma^{*}\right)\left(l^{*}+\gamma^{*}+1\right)-(l+\gamma)(l+\gamma+1)}{y^{2}} .
$$

Here, the quantity $\gamma^{*}$ is defined by $\gamma^{*}=\left(d^{*}-3\right) / 2$. The quantities $n^{*}$ and $l^{*}$ are defined as

$$
\begin{aligned}
n^{*} & \equiv n_{s}-\delta=n+i-\delta, \\
l^{*} & =l+i-\delta,
\end{aligned}
$$

where $\delta$ is the quantum defect determining the energy shifts for the generalized Rydberg series and where $i=i(l)$ is an integral-valued function of the angular momentum. In the supersymmetric interpretation for the valence electron of physical atoms, $i(l)$ is the number of filled lower levels with angular momentum $l$. The introduction of $n_{s}$ is 
motivated by the three-dimensional case, where it is equal to the principal quantum number and takes conventional values in the standard spectroscopic notation. It satisfies $n_{s}=n+i$, where $n$ takes the usual values characteristic of the exact Coulomb system. As an example, the $s$ states of the supersymmetric sodium atom in three dimensions have $i(0)=2$, giving $n_{s}=3, n_{s}=4, n_{s}=5$ for the first three levels [13]. The corresponding values of $n$ are $n=1, n=2, n=3$. For the supersymmetric partner of the exact Coulomb system, $i=1$ and so $n_{s}=n+1=n^{\prime}$, consistent with our previous notation for the supersymmetric case.

The first term of Eq. (36) has the effect of shifting the energy levels, while the second term performs a corresponding shift in the angular-momentum barrier. The combined effect of both terms incorporates the desired dimensional shift. With a nonzero quantum defect $\delta(l)$, the effective potential $v_{\text {eff }}(y)$ plays the role of a supersymmetrybreaking potential. The resulting radial equation has analytical solutions given in terms of the usual Coulomb solutions $w_{d, n, l}(y)$ by $w_{d^{*}, n^{*}, l^{*}}(y)$. These solutions exist for $n \geq l+1$, or $n_{s} \geq l+i+1$. Requiring the existence and orthonormalizability of the wave functions restricts $\delta-i$ according to

$$
\delta-i<l+\gamma+1+\frac{1}{2} j
$$

It is convenient to define a dimensionless quantity $a(l)$ by

$$
a(l)=i-\delta+\frac{1}{2} j
$$

The eigenvalues of the differential equation can then be expressed as

$$
\frac{E_{n^{*}, \gamma^{*}}}{E_{0}}=\frac{-1}{4\left(n^{*}+\gamma^{*}\right)^{2}}=\frac{-1}{4(n+\gamma+a)^{2}} \text {. }
$$

In this equation, we have chosen the eigenenergies so that the limiting case with $d=3$ and $i=j=0$ reproduces the Rydberg series (35). For $i, \delta$, and $j$ chosen so that $a=0$, we obtain the bosonic equation of the Coulomb system discussed in section IIE, up to an energy shift. If $a=1$, the fermionic sector of the Coulomb problem is generated instead. Moreover, the supersymmetric partner of the fermionic sector is generated by setting $a=2$, and each successive iteration of the supersymmetry increments $a$ by one unit. 
A useful stack correspondence can be established between the spectrum of the bosonic sector of the supersymmetric Coulomb system and the SQDT Coulomb spectrum. The map is given by making the following replacements in $w_{d, n, l}^{+}$:

$$
\begin{aligned}
& d \longmapsto d^{*}=d+j, \\
& n \quad n^{*}=n+i-\delta=n_{s}-\delta, \\
& l \quad l^{*}=l+i-\delta .
\end{aligned}
$$

\section{IIIB. Generalized SQDT for the Oscillator System}

The techniques of section IIIA can also be applied to the radial oscillator system in $D$ dimensions. For fixed angular momentum $L$, we can obtain an effective potential $V_{\text {eff }}(Y)$ to be added to Eq. (8) that maintains analytical eigenfunctions while inducing

an integral shift to a new dimension $D^{*} \equiv D+J \geq 1$ and simultaneously modifying the oscillator energy eigenvalues via a shift to a new principal quantum number $N^{*}$. We refer to the resulting theory as the oscillator SQDT.

The appropriate choice of effective potential is

$$
V_{\mathrm{eff}}(Y)=2\left(N-N^{*}+\Gamma-\Gamma^{*}\right)+\frac{\left(L^{*}+\Gamma^{*}\right)\left(L^{*}+\Gamma^{*}+1\right)-(L+\Gamma)(L+\Gamma+1)}{Y^{2}},
$$

where $\Gamma^{*}=\left(D^{*}-3\right) / 2$ and the shifted quantum numbers are given by

$$
\begin{aligned}
N^{*} & \equiv N_{s}-I-\Delta \equiv N+I-\Delta, \\
L^{*} & =L+I-\Delta .
\end{aligned}
$$

Here, $I=I(L)$ is an integral-valued function, analogous to $i(l)$ in the Coulomb case, that can be interpreted as the number of inaccessible lower levels. The quantity $\Delta(N, L)$ is the oscillator equivalent of the Rydberg quantum defect $\delta(n, l)$, modifying the radial-repulsion term in the differential equation. For simplicity in what follows, we take $\Delta$ to depend only on $L$, thereby paralleling the case of alkali-metal atoms for which $\delta$ depends only on $l$. We have also defined a quantity $N_{s}$ playing the role of the principal quantum number in the spectroscopic notation, given by $N_{s}=N+2 I$. If the dimension is unmodified and $\Delta=0$, the choice $I=1$ yields the fermionic sector of the supersymmetry discussed in section IIF. In this limit $N^{\prime}=N^{*}=N+1 \neq N_{s}$, which 
differs from the supersymmetric limit of the Coulomb SQDT where $n^{\prime}=n^{*}=n_{s}$. With our definitions, degenerate levels in the bosonic and fermionic sectors have values of $N$ differing by $2 I$ units, but have the same value of $N_{s}$.

The first term in Eq. (43) implements the eigenenergy shift to the oscillator analogue of the Rydberg series, while the second term is the corresponding anharmonic modification to the potential that maintains analytical eigensolutions. The eigenfunctions solving the resulting effective radial equation are given in terms of the oscillator wave functions $W_{D N, L},(Y)$ of Eq. (9) by $W_{D^{*}, N^{*}, L^{*}}(Y)$. The existence of these solutions requires that the principal quantum number takes the values $N_{s}=L+2 I$, $L+2 I+2, L+2 I+4, \ldots$, or $N=L, L+2, L+4, \ldots$ Requiring orthonormalizability of the wave functions restricts the range of $\Delta-I$ to

$$
\Delta-I<L+\Gamma+\frac{3}{2}+\frac{1}{2} J
$$

We can again introduce a useful dimensionless quantity $A(L)$ by

$$
A(L)=I-\Delta+\frac{1}{2} J
$$

The eigenvalues of the differential equation can be expressed as

$$
\frac{F_{N^{*}, \Gamma^{*}}}{F_{0}}=2 N^{*}+2 \Gamma^{*}+2 A+3=2 N+2 \Gamma+4 A+3
$$

We have chosen the ground-state eigenenergy in analogy with the Coulomb case (41). The extra factor of $2 A$ appears to ensure that the bosonic and fermionic spectra of the limiting supersymmetric case with $\Delta=0, J=0$ have the characteristic degenerate pairing. If $I, \Delta$, and $J$ are selected so that $A=0$, then this SQDT system reduces to the bosonic oscillator discussed in section IIF, up to an energy shift. If $A=1$, it reduces instead to the fermionic partner. If $A=2$, the second-fermionic sector of the supersymmetric oscillator is produced. Each further iteration of supersymmetry produces an additional unit increment of $A$. Note that for fixed $L$ the spacing between successive eigenvalues is always four units, regardless of the value of $A$.

A correspondence can be established between the oscillator SQDT and the bosonic sector of the supersymmetric oscillator. The images of the wave functions are obtained 
by making the replacements:

$$
\begin{aligned}
& D \quad \longmapsto \quad D^{*}=D+J, \\
& N \quad \longmapsto \quad N^{*}=N+I-\Delta, \\
& L \quad \longmapsto \quad L^{*}=L+I-\Delta .
\end{aligned}
$$

\section{Mappings between Bound States of the Coulomb and Oscillator SQDT}

Composition of the mappings in sections IIC, IIIA and IIIB allows us to establish a correspondence between the $d^{*}$-dimensional Coulomb SQDT and the $D^{*}$-dimensional oscillator SQDT. This mapping is described in section IVA. One of its striking features is that the odd-dimensional oscillator can be imaged. In section IVB, we illustrate the mapping with examples involving the three-dimensional Coulomb and oscillator systems.

\section{IVA. The General Case}

The general mapping is given by

$$
\begin{aligned}
W_{D^{*}, N^{*}, L^{*}}(Y) & =K_{d^{*}, n^{*}, \lambda-\frac{1}{2} J+j} Y^{-\frac{1}{2}} w_{d^{*}, n^{*}, l^{*}}\left(\left(n^{*}+\gamma^{*}\right) Y^{2}\right) \\
Y^{2} & =y /\left(n^{*}+\gamma^{*}\right) \\
D^{*} & =2 d^{*}-2-2 \lambda+J-2 j, \\
N^{*} & =2 n^{*}-2+\lambda-\frac{1}{2} J+j \\
L^{*} & =2 l^{*}+\lambda-\frac{1}{2} J+j \\
A & =2 a .
\end{aligned}
$$

This mapping, like the Coulomb-oscillator case discussed in section IIC, is based on a quadratic relationship between the radial variables of the two systems. The constant $K$ can be chosen to preserve the normalization of the wave functions, in which case it has the functional form given in Eq. (13). Note that Eq. (53) is equivalent to a generalization of Eq. (15), given by

$$
\frac{F_{N^{*}, \Gamma^{*}}}{F_{0}}=2 \sqrt{\frac{E_{0}}{-E_{n^{*}, \gamma^{*}}}}+4 a .
$$


Note also that the allowed ranges of the quantum defects given in Eq. (39) and Eq. (46) are compatible with Eq. (55), which guarantees that the image of any orthonormalizable Coulomb radial system is an orthonormalizable oscillator.

To gain insight about the flexibility of this mapping, consider the choice $j=0$. Then, $D^{*}$ lies in the range $1+J \leq D^{*} \leq 2 d-2+J$ with allowed values separated by two units. Since we require $J \geq 1-D$, any $D^{*} \geq 1$ is possible. Moreover, $d$ may take any value greater than one. The above general mapping therefore relates any Coulomb dimension $d>1$ to any oscillator dimension $D^{*} \geq 1$. In particular, Eq. (52) shows that $D^{*}$ is odd if $J$ is chosen to be an odd integer. This is in striking contrast to the usual restriction of $D$ to even values only, as given by Eq. (14).

The Coulomb-oscillator mappings defined earlier are special cases of our general mapping. The correspondence of section IIC is recovered by setting $i=\delta=j=0$ in the Coulomb system and $I=\Delta=J=0$ in the oscillator system, so that $A=2 a=0$. Figure 2 is a commutative diagram showing the relationship between this simpler mapping and the general mapping. Similarly, the mapping of section IIF between the fermionic sector of the supersymmetric Coulomb system and the second-fermionic sector of the supersymmetric oscillator is reproduced with the choices $i=1, \delta=0$, $j=0$ and $I=2, \Delta=0, J=0$, so that $A=2 a=2$.

For the case of constant nonnegative integral $A$ and $a$, Eq. (55) controls the relationship between the supersymmetric sectors of the two systems. While any iteration of the supersymmetry for the Coulomb system can be taken, only even iterations of the oscillator supersymmetry appear. This is why we introduced the second-fermionic sector of the supersymmetric oscillator in section IIF. It is therefore possible to combine Figures 1 and 2 in a single commutative diagram. Moreover, the general mapping shows that Figure 1 can be extended downward to incorporate higher iterations of the supersymmetry. The result is an infinite series of mappings relating Coulomb systems with $a=2,3,4, \ldots$ to oscillator systems with $A=4,6,8, \ldots$, respectively.

\section{IVB. Three-Dimensional Coulomb and Oscillator Systems}

To obtain further insight about the content of the general mapping of section 
IVA, we next restrict attention to the special case where both systems are threedimensional. Since this choice can be implemented with $j=0$, we assume this in what follows.

The general mapping becomes

$$
\begin{aligned}
W_{3, N^{*}, L^{*}}(Y) & =K_{3, n^{*}, \frac{1}{2}} Y^{-\frac{1}{2}} w_{3, n^{*}, l^{*}}\left(n^{*} Y^{2}\right), \\
N^{*} & =2 n^{*}-\frac{3}{2} \\
L^{*} & =2 l^{*}+\frac{1}{2} \\
\Delta-I & =2(\delta-i)+\lambda-\frac{1}{2} .
\end{aligned}
$$

The values of $\lambda$ allowed by Eq. (54) are $\lambda=0,1$. The orthonormality requirements Eq. (39) and Eq. (46) become

$$
\begin{aligned}
\delta-i & <l+1 \\
\Delta-I & <L+\frac{3}{2} .
\end{aligned}
$$

We can regard Eq. (61) and Eq. (62) as conditions limiting the choice of quantum defects in the two systems to a semi-infinite region of the $(\Delta-I)$ versus $(\delta-i)$ plane. The condition (60) then further restricts the choice to a straight line in this region.

One interesting special case is obtained by requiring that the oscillator be exact in the sense that $\Delta-I=0$. Then, Eq. (60) becomes

$$
\delta-i=-a=\frac{1}{2}\left(\frac{1}{2}-\lambda\right),
$$

showing that a nonzero defect in the Coulomb system is necessary. The eigenvalues of the equations are

$$
\begin{aligned}
E_{n^{*}, \gamma^{*}} & =\frac{-E_{0}}{\left(2 n+\lambda-\frac{1}{2}\right)^{2}}, \\
F_{N, \Gamma} & =F_{0}(2 N+2 \lambda+2),
\end{aligned}
$$

and the relationships among the principal quantum numbers and the angular momenta become

$$
\begin{aligned}
L & =2 l^{*}+\frac{1}{2}, \\
N & =2 n^{*}-\frac{3}{2} .
\end{aligned}
$$


Selecting $\lambda=1$ for definiteness, we see that Eq. (66) maps each successive Coulomb angular momentum $l^{*}=\frac{1}{4}, l^{*}=\frac{5}{4}, l^{*}=\frac{9}{4}, \ldots$ to every second oscillator angular momentum starting at $L=\lambda: L=1, L=3, L=5, \ldots$ The mapping therefore preserves the degeneracy of states. For instance, the kets $\left|n^{*}=\frac{9}{4}, l^{*}=\frac{1}{4}\right\rangle$ and $\left|n^{*}=\frac{9}{4}, l^{*}=\frac{5}{4}\right\rangle$, which are degenerate in the Coulomb system, are mapped to the degenerate states $|N=3, L=1\rangle$ and $|N=3, L=3\rangle$ in the oscillator system. This feature is also a characteristic of the original mapping of section IIC. The main differences here are that the Coulomb effective angular momenta $l^{*}$ are nonintegral and, more importantly, that both systems are three dimensional.

A second case of interest is obtained when the Coulomb system is exact, i.e., $\delta-i=0$. The condition $j=0$ implies that $a=0$ too. Then, Eq. (55) becomes $\Delta-I=\lambda-\frac{1}{2}$, showing that a nonzero defect is again needed, this time in the oscillator system. The eigenvalues are

$$
\begin{aligned}
E_{n, \gamma} & =\frac{-E_{0}}{4 n^{2}}, \\
F_{N^{*}, \Gamma^{*}} & =F_{0}(2 N-2 \lambda+4),
\end{aligned}
$$

and the mapping gives

$$
\begin{aligned}
L^{*} & =2 l+\frac{1}{2}, \\
N^{*} & =2 n-\frac{3}{2} .
\end{aligned}
$$

The first of these equations shows every second oscillator angular momentum is imaged, which again preserves the degeneracy of states. If, for example, we choose $\lambda=0$, then the degenerate Coulomb states $|n=3, l=1\rangle$ and $|n=3, l=2\rangle$ map to the degenerate oscillator states $\left|N^{*}=\frac{9}{2}, L^{*}=\frac{5}{2}\right\rangle$ and $\left|N^{*}=\frac{9}{2}, L^{*}=\frac{9}{2}\right\rangle$.

In the above examples, the quantities $\Delta-I$ and $\delta-i$ are constant. In physical systems such as alkali-metal atoms, $\delta-i$ depends on $l$ and tends towards zero as $l$ increases. This feature can also be incorporated in our general mapping. It implies a dependence of $\Delta-I$ on $L$, which might reflect a realistic feature of a physical oscillator such as a cloud of particles caught in a Penning trap.

As an example, we map the SQDT radial equation for the physical sodium atom into a three-dimensional SQDT oscillator. In sodium, the inaccessibility of the levels 
occupied by the ten inner electrons is implemented by the choices $i(0)=2, i(1)=1$, and $i(l \geq 2)=0$. The quantum defects $\delta(l)$ in this case are known [22]. Choosing for definiteness $\lambda=1$ and selecting $I(0)=2, I(1)=1, I(L \geq 2)=0$, the values of $\Delta$ can be found from Eq. (60). Table 1 lists the results. As expected, the values of $\Delta$ tend towards $\frac{1}{2}$ as $L$ increases.

\section{Mappings for Continuum States}

In previous sections, we have explored mappings between the bound states of the Coulomb and oscillator systems. It is natural to consider whether similar mappings exist taking the unbound Coulomb states into an appropriate oscillator. This question is of lesser physical interest at present, so we restrict ourselves here to a brief outline of a possible approach to this issue.

The Coulomb problem with energies $E>0$ can be viewed as a scattering problem. Following the general procedure of section IIA again yields the differential equation (2), but with $E>0$. In terms of the confluent hypergeometric function ${ }_{1} F_{1}$, the solutions are

$$
\begin{aligned}
w_{d, E, l}(y) \propto & y^{l+\gamma+1} \exp \left( \pm i y \sqrt{\frac{E}{E_{0}}}\right) \\
& \times{ }_{1} F_{1}\left(l+\gamma+1 \pm \frac{1}{2 i} \sqrt{\frac{E_{0}}{E}}, 2(l+\gamma+1), \mp 2 i y \sqrt{\frac{E}{E_{0}}}\right) .
\end{aligned}
$$

The upper and lower signs correspond to outgoing and incoming waves, respectively. The results of section IIA can be recovered by taking $E$ to be negative and choosing the upper sign.

It turns out that the appropriate image oscillator system [23] is the inverted os-

cillator, with potential $U(R)=-\frac{1}{2} M \Omega R^{2}$. This system is unbound. The procedure of section IIB gives a differential equation identical to Eq. (8) except that the sign of the potential $Y^{2}$ is reversed. The solutions $W_{D, F, L}(Y)$ are

$$
W_{D, F, L}(Y) \propto Y^{L+\Gamma+1} \exp \left( \pm \frac{1}{2} i Y^{2}\right){ }_{1} F_{1}\left(\frac{1}{2}\left(L+\Gamma+\frac{3}{2}\right) \mp \frac{i F}{4 F_{0}}, L+\Gamma+\frac{3}{2}, \mp i Y^{2}\right)
$$

These functions may not be physically permissible, but are relevant for the purposes 
of establishing a mapping. With the choice of the upper sign, the wave functions for the usual oscillator may be obtained up to a constant by the analytic continuation $Y^{2} \rightarrow i Y^{2}$ and $F \rightarrow-i F$.

A correspondence analogous to the mapping of section IIC exists between the continuum Coulomb states and the inverted-oscillator functions. It is

$$
\begin{aligned}
W_{D, F, L}(Y) & \propto Y^{-\frac{1}{2}} w_{d, E, l}\left(\frac{Y^{2}}{2 \sqrt{E / E_{0}}}\right), \\
Y^{2} & =2 y \sqrt{E / E_{0}}, \\
D & =2 d-2-2 \lambda, \\
L & =2 l+\lambda \\
\frac{F}{F_{0}} & =2 \sqrt{\frac{E_{0}}{E}} .
\end{aligned}
$$

There are many similarities between this mapping and the one discussed in section IIC. Again, $\lambda$ must be integral, so only even-dimensional oscillators are available as images. Also, the angular momenta $L$ are restricted to being either all odd or all even, thus eliminating half the oscillator states. However, the energy relation (78), unlike (15), involves continuous values of $E$ and $F$. It also reveals that negative energies $F$ are excluded from the mapping. As one energy tends to zero the other tends to infinity.

As an aside, we remark that the negative energies $F$ do appear when considering the repulsive Coulomb problem. The differential equation of this problem is mapped into the inverted-oscillator differential equation [24] by a map with (74) through (77) unchanged but with a negative sign taken for the square root in Eq. (78).

Although this lies outside the scope of the present work, it seems feasible that supersymmetry could be introduced into these systems along with the corresponding SQDT. We conjecture that this allows for odd dimensions $D$. Since parabolic coordinates have some advantages for scattering problems, it would also be interesting to perform an analysis in terms of the dual parabolic-coordinate supersymmetries of ref. [25] instead of the spherical-coordinate supersymmetry used here. 


\section{Summary}

In this paper, we generalized the radial mappings first identified by Schrödinger that relate the Coulomb and oscillator systems. Our principal result is a mapping between the supersymmetry-based quantum-defect theories for the Coulomb and oscillator systems in arbitrary dimensions. In particular, odd oscillator dimensions can be accessed as well as the usual even ones. The mapping and some of its limits are illustrated in Figures 1 and 2.

In deriving this result, we have extended to arbitrary dimensions the analytical SQDT in three dimensions used to describe physical alkali-metal atoms. An analogous SQDT for the harmonic oscillator in arbitrary dimensions has also been presented. In suitable limits, these theories reproduce the bosonic and fermionic sectors of the corresponding supersymmetric quantum-mechanical systems. We have elucidated a basic relationship between the supersymmetric radial Coulomb and oscillator systems: the $q$ th iteration of supersymmetry for the Coulomb system corresponds naturally to the $2 q$ th iteration of supersymmetry for the oscillator. For the special case of the onedimensional radial oscillator, we uncovered a quantum-mechanical supersymmetry in which the parity is restricted to be either odd or even. We have also briefly considered mappings relating the continuum-spectrum states of the Coulomb and oscillator systems.

The issue of the physical relevance of our results has also been addressed in part. The three-dimensional Coulomb SQDT is known to provide a good analytical description of the behaviour of Rydberg atoms. Our mapping provides a means of obtaining an equivalent analytical oscillator SQDT. An explicit example mapping the sodium atom to an oscillator SQDT is given in Table 1. It is also possible that an oscillator SQDT could be used to describe a suitable physical system, perhaps the Penning trap. If this can be realized in practice, the generalized mapping presented here could provide a connection between two apparently disparate physical systems.

\section{Acknowledgments}

We thank Larry Biedenharn for discussion. This work was supported in part by 
the United States Department of Energy under grant number DE-FG02-91ER40661.

\section{References}

1. T. Levi-Civita, Act. Math. 30, 305 (1906)

2. E. Schrödinger, Proc. Roy. Irish Acad. Sect. A 46, 183 (1941)

3. J.D. Louck, J. Mol. Spectr. 4, 334 (1960)

4. D. Bergmann and Y. Frishman, J. Math. Phys. 6, 1855 (1965)

5. V.A. Dulock and H.V. McIntosh, Pacific J. Math. 19, 39 (1966)

6. R. Rockmore, Am. J. Phys. 43, 29 (1975)

7. E. Chacón, D. Levi and M. Moshinsky, J. Math. Phys. 17, 1919 (1976)

8. J. Č́źžek and J. Paldus, Internat. J. Quantum Chem. 12, 875 (1977)

9. V.A. Kostelecký, M.M. Nieto and D.R.Truax, Phys. Rev. D 32, 2627 (1985)

10. V.A. Kostelecký, p. 295 in Symmetries in Science VII, eds. B. Gruber and T. Otsuka (Plenum, New York, 1994) quant-ph/9508015)

11. H. Nicolai, J. Phys. A 9, 1497 (1976)

12. E. Witten, Nucl. Phys. B185, 513 (1981)

13. V.A. Kostelecký and M.M. Nieto, Phys. Rev. Lett. 53, 2285 (1984); Phys. Rev. A 32, 1293 (1985).

14. V.A. Kostelecký and M.M. Nieto, Phys. Rev. A 32, 3243 (1985). For a review and references to related literature, see, for example, ref. [10]. Recent developments in the subject are discussed in R. Bluhm and V.A. Kostelecký, Phys. Rev. A 49, 4628 (1994)(quant-ph/9508020); ibid., 50, R4445 (1994) (hepph/9410325); ibid., 51, 4767 (1995) quant-ph/9506009); R. Bluhm, V.A. Kostelecký and B. Tudose, Phys. Rev. A 52, 2234 (1995)(quant-ph/9509010); ibid., in press (quant-ph/9510023).

15. P. Kustaanheimo and E. Stiefel, J. Reine Angew. Math. 218, 204 (1965)

16. A. Cisneros and H.V. McIntosh, J. Math. Phys. 10, 277 (1969)

17. M. Boiteux, Physica 65, 381 (1973) 
18. A.C. Chen, Phys. Rev. A 22, 333 (1980); 2901; J. Math. Phys. 23, 412 (1982)

19. M. Kibler and T. Negadi, Lett. Nuov. Cim. 37, 225 (1983)

20. L.S. Davytan, L.G. Mardoyan, G.S. Pogosyan, A.N. Sissakian and V.M. Ter-Antonyan, J. Phys. A 20, 6121 (1987)

21. D. Lambert and M. Kibler, J. Phys. A 21, 307 (1988)

22. H.G. Kuhn, Atomic Spectra (Academic, New York 1969)

23. A.O. Barut, C.K.E. Schneider and R. Wilson, J. Math. Phys. 20, 2244 (1979)

24. P. Collas, J. Math. Phys. 22, 2512 (1991)

25. R. Bluhm and V.A. Kostelecký, Phys. Rev. A 47, 794 (1993) 
Table 1. Possible parameters for a mapping between the radial Coulomb and oscillator systems in three dimensions. For the choices $j=0$ and $\lambda=1$, values of $l, i, n, n_{s}$, and $\delta$ are tabulated for sodium along with the corresponding values of $L, I, N, N_{s}$, and $\Delta$ under the mapping (60). The quantities $I(L)$ have been selected to fill all levels below $N_{s}=5$.

Figure 1. Supersymmetric mappings. Relationships are shown interconnecting the bosonic and fermionic partners of the Coulomb and oscillator systems. The diagram is commutative.

Figure 2. SQDT mappings. Relationships are shown interconnecting the bosonic and SQDT sectors of the Coulomb and oscillator systems. The diagram is commutative. 


\begin{tabular}{|r|l|l|l|l||r|l|l|l|l|}
\hline \multicolumn{4}{|c||}{ Coulomb system (sodium) } & \multicolumn{5}{|c|}{ Oscillator system } \\
\hline$l$ & $i$ & \multicolumn{1}{|c|}{$n$} & \multicolumn{1}{|c|}{$n_{s}$} & \multicolumn{1}{c|}{$\delta$} & $L$ & $I$ & \multicolumn{1}{c|}{$N$} & $N_{s}$ & \multicolumn{1}{|c|}{$\Delta$} \\
\hline 0 & 2 & $\geq 1$ & $\geq 3$ & 1.35 & 1 & 2 & $\geq 1$ & $\geq 5$ & 1.20 \\
1 & 1 & $\geq 2$ & $\geq 3$ & 0.859 & 3 & 1 & $\geq 3$ & $\geq 5$ & 1.218 \\
2 & 0 & $\geq 3$ & $\geq 3$ & 0.01 & 5 & 0 & $\geq 5$ & $\geq 5$ & 0.52 \\
3 & 0 & $\geq 4$ & $\geq 4$ & 0.00 & 7 & 0 & $\geq 7$ & $\geq 7$ & 0.50 \\
$\geq 4$ & 0 & $\geq l+1$ & $\geq l+1$ & 0 & $\geq 9$ & 0 & $\geq L$ & $\geq L$ & 0.5 \\
\hline
\end{tabular}

Table 1 


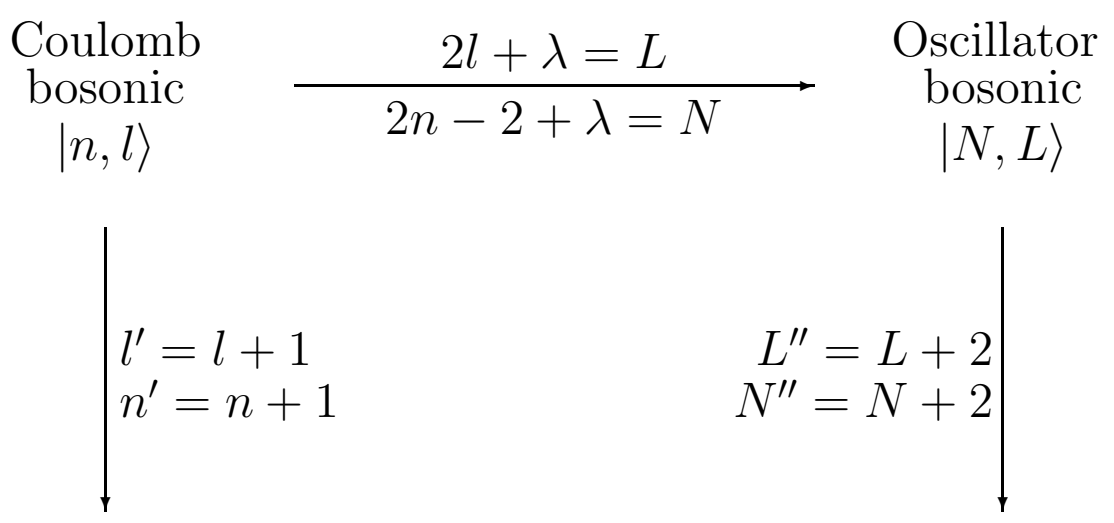

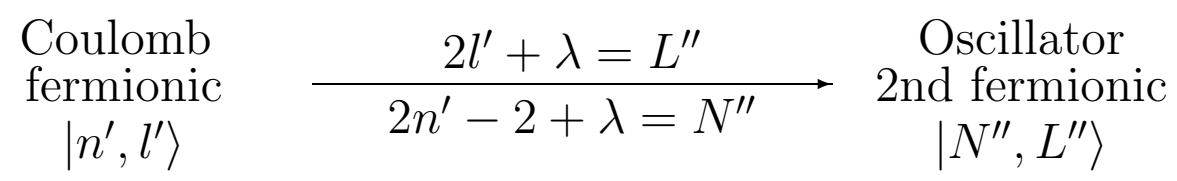

Figure 1 


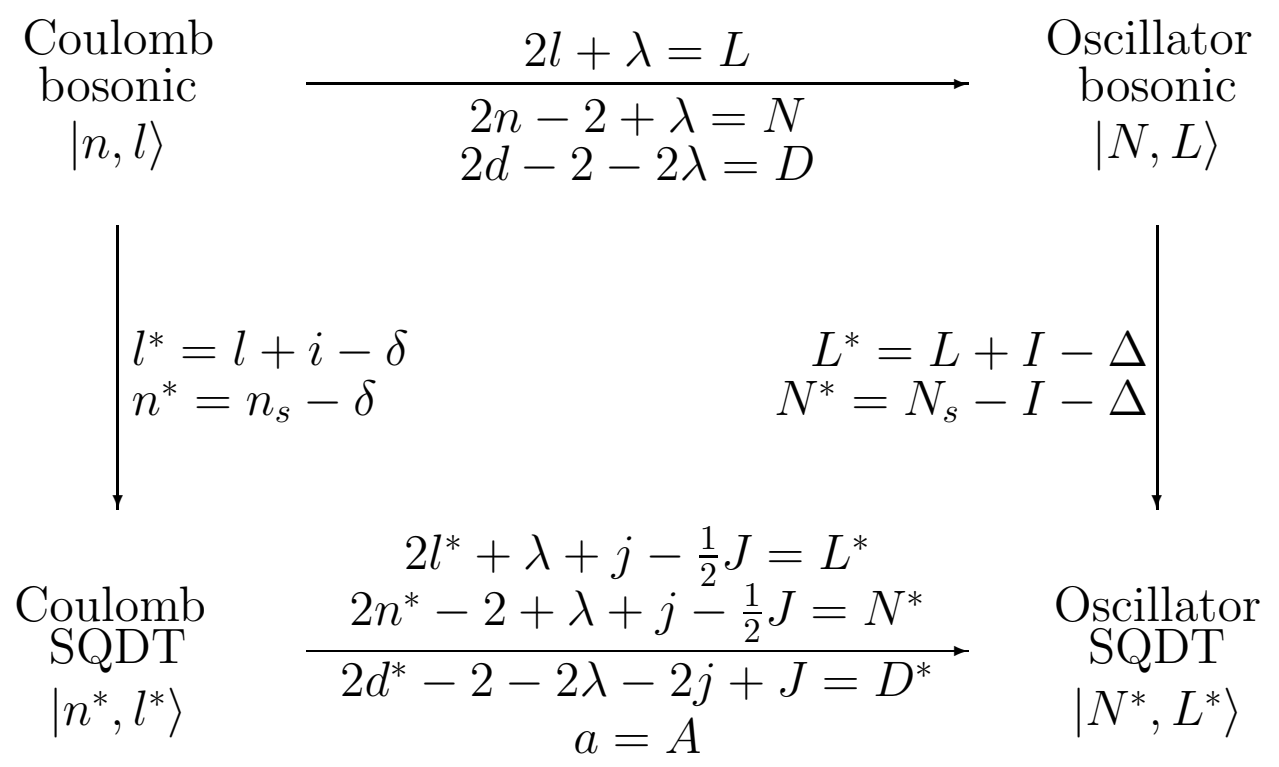

Figure 2 